Gefässchirurgie 2021 $26: 298-307$ https://doi.org/10.1007/s00772-021-00762-4 Angenommen: 23. Dezember 2020 Online publiziert: 18. März 2021

(c) Der/die Autor(en) 2021

\section{Einleitung}

Das Nahtaneurysma (NA) ist eine Komplikation einer gefäßchirurgischen Rekonstruktion und gehört definitionsgemäß zum falschen Aneurysma (Aneurysma spurium/falsum). In der Pionierzeit der Gefäßchirurgie waren die NA mit 3-24\% eine häufige postoperative Komplikation [3]. Als Nahtmaterial wurde Seide verwendet, welches nicht selten zur Nahtruptur führte. NA entwickeln sich im Allgemeinen langsam und führen zur partiellen oder kompletten Ruptur der Nahtreihe [4]. Im Zusammenhang mit NA sind echte Aneurysmen (Dilatation der Arterie oder des Gefäßersatzmaterials) und falsche Aneurysmen, z.B. an der Naht oder Gefäßwandalteration, die zu einer Naht- oder Graftruptur führen können, voneinander abzugrenzen.

Als Besonderheit kann es bei Dilatation des Transplantats im Sinne eines echten Aneurysmas im Verlauf zur Graftruptur mit Entwicklung eines falschen Aneurysmas kommen.

Differenzialdiagnostisch sind eine infektbedingte Nahtruptur sowie eine falsche Kaliberadaptation bei End-zuSeit-Anastomose zu unterscheiden [5, 29].

Nach autologem Gefäßersatz oder einer Direktnaht sind falsche Aneurysmen selten, da eine komplette Endothelialisierung der Naht mit schmaler Narbenbildung entsteht [15]. Die Verwendung alloplastischen Materials führt $\mathrm{zu}$ einem „compliance mismatch“, was den

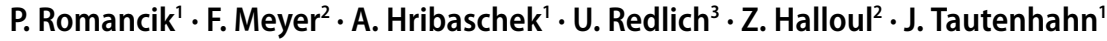

'Klinik für Gefäßchirurgie, Klinikum Magdeburg, Magdeburg, Deutschland

${ }^{2}$ Arbeitsbereich Gefäßchirurgie, Klinik für Allgemein-, Viszeral-, Gefäß- und Transplantationschirurgie, Universitätsklinikum Magdeburg, Magdeburg, Deutschland

${ }^{3}$ Institut für Diagnostische und Interventionelle Radiologie, Klinikum Magdeburg, Magdeburg, Deutschland

\title{
Ergebnisse nach gefäßchirurgischer Versorgung von Leistennahtaneurysmen
}

„shear stress" im Bereich der Anastomose erhöht und die arterielle Wand verletzt [25]. Bei unterschiedlicher Elastizität des Gefäßersatzes/der Wirtsarterie und ausbleibender Vernarbung der Nahtreihe ist zusätzlich das Nahtmaterial, dessen Verankerung und Materialqualität lebenslang für die Stabilität der Anastomose entscheidend [15]. Im Progress des NA kommt es strömungsbedingt zur zunehmenden Wandthrombosierung. Die Kompression benachbarter Strukturen, die komplette Thrombosierung, Embolisation und Ruptur sind weitere Komplikationen. Im Leistenbereich besteht eine Indikation zur Sanierung ab 2-2,5 cm Durchmesser, in den anderen Anastomosengebieten besteht eine Indikation zur Sanierung bereits bei Bestätigung der Diagnose, um spätere Komplikationen zu vermeiden [14, 20].

Das Ziel der Studie bestand darin, anhand von konsekutiven Patienten eines zertifizierten Gefäßzentrums über einen definierten Zeitraum das Auftreten von Leisten-NA als Spätkomplikation im Rahmen einer retrospektiven Fallserie $\mathrm{zu}$ untersuchen und der medizinischwissenschaftlichen Literatur gegenüberzustellen.

\section{Material und Methoden}

Über einen definierten Beobachtungszeitraum wurden in der Klinik für Gefäßchirurgie des Klinikums Magdeburg alle konsekutiven Patienten mit einem Leisten-NA nach gefäßchirurgischen Ope- rationen in einem computerbasierten Register im Rahmen dieser retrospektiven unizentrischen Observationsstudie zur gefäßchirurgischen Qualitätssicherung in der Reflexion des klinischen Alltags (Studiendesign) erfasst, die operativ behandelt worden waren. Im gesamten Patientenkollektiv (NA-Gruppe) wurden zwei Untersuchungsgruppen gebildet. Die erste Gruppe (NA-ohneRezidiv-Gruppe = NAOR-Gruppe) umfasste Patienten, bei denen es in diesem Zeitraum nicht zum NA-Rezidiv gekommen war. In der zweiten Gruppe (NAmit-Rezidiv-Gruppe $=$ NAMR-Gruppe) befanden sich Patienten, die im angegebenen Zeitraum ein Rezidiv entwickelt und einer Sanierung bedurft hatten.

Die Diagnostik der NA erfolgte mittels Duplexsonographie, computertomographischer Angiographie (CTA) und Magnetresonanzangiographie (MRA). Bei den Leisten-NA, die mittels intraarterieller digitaler Subtraktionsangiographie (DSA) diagnostiziert wurden, handelte es sich um Zufallsbefunde im Rahmen einer interventionellen Versorgung der Begleitbefunde. Bei Auswertung des Durchmessers wurde die laterolaterale Messung verwendet. Anamnestische und patientenbezogene Daten wurden aus der Patientenakte, der Klinikdatenbank und der Dokumentation der poststationären Untersuchungen entnommen. Es fanden demografische, anamnestische und klinische Daten wie Alter und Geschlecht der Patienten, vorausgegangene Gefäßoperationen, Symptomatik und postopera- 
Tab. 1 Komorbiditäten und Risikofaktoren bei Nahtaneurysmen (NA)

\begin{tabular}{|c|c|}
\hline $\begin{array}{l}\text { Diagnose- } \\
\text { gruppe }\end{array}$ & Entität \\
\hline $\begin{array}{l}\text { Kardiozirkula- } \\
\text { torisch }\end{array}$ & $\begin{array}{l}\text { Arterielle Hypertonie (aHT), koronare Herzerkrankung (KHK), akuter Myokardin- } \\
\text { farkt (AMI), zerebraler Insult, aneurysmatische Erkrankung }\end{array}$ \\
\hline $\begin{array}{l}\text { Pulmonal/ } \\
\text { pulmologisch }\end{array}$ & Chronisch-obstruktive Lungenerkrankung (COPD) \\
\hline Metabolisch & Hyperurikämie, Diabetes mellitus (DM), Hyperlipidämie (HLP), Adipositas \\
\hline $\begin{array}{l}\text { Gerinnungs- } \\
\text { inhibition }\end{array}$ & $\begin{array}{l}\text { Orale Antikoagulation (OAK: Vitamin-K-Antagonisten und neue Antikoagulanti- } \\
\text { en) }\end{array}$ \\
\hline Übrige & $\begin{array}{l}\text { Nikotinabusus, Alkoholabusus, onkologische Erkrankung (ONK), Hernienerkran- } \\
\text { kung }\end{array}$ \\
\hline
\end{tabular}

tive Komplikationen Berücksichtigung. Des Weiteren erfolgte die spezifisch befundbezogene Datenerhebung von: Durchmesser, Rekonstruktionsmethode (Rhaphie \pm Patch, Interponat), Zeitintervall von der primären Operation (OP) bis zur Sanierung des NA und gegebenenfalls bis zur Entwicklung eines NARezidivs, Offenheitsrate, ob eine lokale Desobliteration (TEA) im OP-Gebiet bereits durchgeführt wurde und ob eine Infektion vorhanden war. Weiterhin erfolgte eine Auswertung von Komplikationen nach NA-Versorgung auch hinsichtlich der Indikationsstellung.

Komorbiditäten und Risikofaktoren der vorliegenden Analyse stellt • Tab. 1 dar.

Das frühpostoperative Ergebnis wurde mit der operativen Morbidität (im Besonderen durch allgemeine und spezifische Komplikationsrate) und Letalität charakterisiert.

Die Offenheitsrate der Leistenrekonstruktion wurde im Rahmen der postoperativen Nachsorge mittels Doppler-/ Duplexsonographie verifiziert. Eine Rekonstruktion war als primär offen klassifiziert, wenn keine weitere Operation oder Intervention nötig war, um die Funktionalität der Rekonstruktion zu gewährleisten.

\section{Statistik}

Die Arbeit beschreibt stetige Variablen wie Alter oder Zeitintervall mit den üblichen Maßzahlen, Mittelwert, Standardabweichung, Minimum und Maximum. Kategoriale Variablen wie zum Beispiel Geschlecht oder Voroperationen charakterisieren absolute und relative Häufigkeiten. Die Prüfung auf
Unabhängigkeit zwischen kategorialen Variablen verwendet den $\chi^{2}$-Test. Die Analyse, ob es einen systematischen Unterschied zwischen zwei Gruppen, z.B. Geschlecht hinsichtlich einer stetigen Variable (Alter) gibt, verwendete bei unabhängigen Stichproben den MannWhitney-U-Test und bei verbundenen Stichproben den Wilcoxon-Test. Bei mehr als zwei Gruppen, z.B. bei Durchführung einer Leisten-TEA (ja, nein, unbekannt) versus Zeitintervall von der primären Operation bis zur NA-Entwicklung, wurde bei unabhängigen Stichproben der Kruskal-Wallis-Test genutzt. Die Beziehung zwischen Gefäßrekonstruktion und Rezidiventwicklung bei NA in der Leiste wurde mittels logistischer Regressionsanalyse (Odds Ratios mit 95\%-Konfidenzintervallen) untersucht. Die Bestimmung der Durchgängigkeitsrate der Gefäßrekonstruktion erfolgte durch Kaplan-Meier-Schätzung. Des Weiteren wird die mediane Überlebenszeit sowie das dazugehörige 95\%Konfidenzintervall berechnet. Die Darstellung des Auftretens der Leisten-NA erfolgte mittels Eins-minus-Überleben. Um die Nullhypothese verwerfen zu können, wurde als statistisch signifikant ein $p$-Wert $<0,05$ angenommen. Bei dem Vergleich der primären Offenheitsrate zwischen Interponaten und Aneurysmorrhaphien in der Leiste wurde der Log-Rank-Test verwendet. Die statistische Analyse erfolgte mit der Software IBM $^{\circledR}$ SPSS $^{\circledR}$ Statistics, Version 24.0 (Copyright 1989-2016, SPSS Inc., Chicago, IL, USA).
Tab. 2 Komorbiditäten und deren Häufig-

keit

\begin{tabular}{lll}
$\begin{array}{l}\text { Nebendiag- } \\
\text { nosen }\end{array}$ & $\begin{array}{l}\text { Anzahl der } \\
\text { Patienten }\end{array}$ & $\begin{array}{l}\text { Angabe } \\
\text { in } \%\end{array}$ \\
\hline aHT & 54 & 79,4 \\
\hline AVK & 54 & 79,4 \\
\hline $\begin{array}{l}\text { Nikotinabusus } \\
\text { KHK }\end{array}$ & 41 & 60,3 \\
\hline COPD & 29 & 42,6 \\
\hline HLP & 21 & 30,9 \\
\hline $\begin{array}{l}\text { Hernien- } \\
\text { erkrankung }\end{array}$ & 13 & 19,1 \\
\hline $\begin{array}{l}\text { Adipositas } \\
\text { Alkoholabusus }\end{array}$ & 11 & 16,2 \\
\hline $\begin{array}{l}\text { OAK } \\
\text { AAA }\end{array}$ & 11 & 16,2 \\
\hline $\begin{array}{l}\text { Onkologische } \\
\text { Erkrankung }\end{array}$ & 8 & 16,2 \\
\hline $\begin{array}{l}\text { DM } \\
\text { Hyperurikämie }\end{array}$ & 7 & 14,7 \\
\hline Apoplex & 2 & 14,7 \\
\hline $\begin{array}{l}\text { CNI } \\
\text { aHT arterielle Hypertonie, AVK arterielle Ver- } \\
\text { schlusskrankheit }\end{array}$ & 11,8 \\
\hline & 1 & 11,8 \\
\hline
\end{tabular}

\section{Erklärung}

Datengenerierung, Dokumentation und Auswertung wurden entsprechend der Erfordernisse der Datenschutzgesetze der Bundesrepublik Deutschland und des Bundeslandes Sachsen-Anhalt vorgenommen. Die Studie wurde entsprechend der Vorgaben der „Declaration of Helsinki for Biomedical Research" von 1964 des „Weltärztebundes“ und ihrer Revisionen und Novellierungen als auch der Instruktionen der institutionellen Ethikkommission sowie nach den Prinzipien der "good clinical (and research) practice" durchgeführt.

Da die studiengegenständlichen Forschungsdaten keinem individuellen $\mathrm{Pa}$ tienten mehr zugeordnet werden können, bestand keine Beratungszuständigkeit der Ethikkommission.

Alle Patienten wurden präoperativ einem adäquaten Aufklärungsgespräch unter Aufführung der operativen Komplikationsmöglichkeiten unterzogen, wonach sie den Aufklärungsbogen unterzeichneten unter Aushändigung einer Kopie, wie angezeigt.

Hinsichtlich der bei der Studienkonzeption zu beachtenden Aspekte „Ge- 
Gefässchirurgie 2021 ·26:298-307 https://doi.org/10.1007/s00772-021-00762-4

(c) Der/die Autor(en) 2021

P. Romancik · F. Meyer · A. Hribaschek · U. Redlich · Z. Halloul · J. Tautenhahn

\section{Ergebnisse nach gefäßchirurgischer Versorgung von Leistennahtaneurysmen}

\section{Zusammenfassung}

Hintergrund. Nahtaneurysmen (NA) sind ein relevanter Aspekt der Sekundärversorgung geworden. Das Ziel der Studie bestand darin, bei Patienten eines zertifizierten Gefäßzentrums das Auftreten von NA im Rahmen einer retrospektiven Fallserie zu untersuchen und selektiv eruierten Referenzen der wissenschaftlichen Literatur gegenüberzustellen.

Patienten und Methode. Über einen definierten Untersuchungszeitraum wurden alle Patienten mit einem Leisten-NA hinsichtlich patientenabhängiger Faktoren (Alter, Geschlechtsverteilung), NA-Größe, chirurgischer Versorgung (Rhaphie \pm Patch vs. Interponat) und Ergebnis (Offenheitsrate, NARezidiv, Morbidität, Letalität) charakterisiert.
Ergebnisse. Die meisten NA traten nach Anlage einer aortobifemoralen Prothese (52,3\%) auf. Das durchschnittliche Zeitintervall bis zur NA-Entwicklung betrug 55,4 Monate. Über die Hälfte der NA ( $n=44 ; 51,2 \%)$ wurden primär mittels Interponat versorgt. - Die Aneurysmorrhaphie mit bzw. ohne Patch hat eine signifikant höhere Wahrscheinlichkeit für ein NA-Rezidiv im Vergleich zum Interponat ( $p=0,026$ bzw. $p=0,006)$. Der durchschnittliche Durchmesser der LeistenNA betrug 46,4 mm. Bei den postoperativen Komplikationen waren am häufigsten nicht operationspflichtige Lymphozelen (25,6\%), Hämatome mit notwendiger Ausräumung $(8,1 \%)$ und Infektionen mit erforderlicher Explantation des alloplastischen Materials
(4,7\%). Die 30-Tage-Letalität betrug 2,3\%. Die notfallmäßig versorgten NA wiesen eine tendenziell höhere Wahrscheinlichkeit einer postoperativen Komplikation $(p=0,09)$ auf. Schlussfolgerungen. Nahtaneurysmen sind mit einer Inzidenz von 0,2-15\% keine seltene Komplikation in der Gefäßchirurgie. Die Interponatanlage ist die Therapie der Wahl bei der Versorgung der Leisten-NA. Alle Patienten sollten über die Wichtigkeit einer lebenslangen gefäßchirurgischen Nachsorge aufgeklärt werden.

Schlüsselwörter

Risikofaktoren · Indikationsstellung · Klinische Ergebnisse $\cdot$ Offenheitsrate $\cdot$ Nachsorge

\section{Results of vascular surgical treatment of inguinal suture aneurysms}

\section{Abstract}

Background. Suture aneurysms (SA) have increasingly become a relevant focus of secondary care.

The aim of the study was to detect the occurrence of SA as a late complication in patients of a certified center of vascular medicine in a retrospective case series and based on operative management experiences as well as selective references from the literature.

Patients and methods. Over a defined observational time period all consecutive patients with inguinal SA were documented in a computer-based data registry as part of this retrospective single center observational study. The cases were characterized by patient-dependent factors (age, sex ratio), SA size, vascular surgical approach (e.g. aneurysmorrhaphy \pm patch vs. interponate) and outcome aspects (patency rate, SA recurrence, morbidity, mortality). Results. The majority of SA occurred after implantation of an aorto-bifemoral prosthesis $(52.3 \%)$ in the groin. The mean time interval to SA manifestation in the groin was 55.4 months. More than half of SA $(n=44 ; 51.2 \%)$ were treated with an interponate-aneurysmorrhaphy \pm patch showed a significantly higher probability for generation of SA compared with an interponate ( $p=0.026$ and $p=0.006$, respectively). The mean diameter of the inguinal SA was $46.4 \mathrm{~mm}$. In the spectrum of postoperative complications, the most frequent problems were lymphocele with no need for surgical intervention (25.6\%), hematoma with need of surgical intervention (8.1\%) and infections with necessary postoperative explantation of alloplastic materials (4.7\%). The 30-day mortality was $2.3 \%$. Emergency operations for SA showed only a tendency to higher probability of postoperative complications $(p=0.09)$.

Conclusion. SA with an incidence of $0.2-15 \%$ can be considered a not uncommon and, thus, noteworthy complication in vascular surgery. Open reconstruction remains the standard procedure for SA at the groin (in particular, implantation of an interponate). Postoperatively, all patients need to be notified on the importance of a regular and life-long vascular surgical follow-up.

Keywords

Risk factors - Indication finding · Outcome . Patency rate - Follow-up care plante Untersuchungen am Menschen, (potenzielle) Gefahren, Datenschutz" ist grundsätzlich festzustellen: Eine (potenzielle) Gefahr bei Studienteilnahme entstand nicht.

\section{Ergebnisse}

Im Beobachtungszeitraum vom 01.04.2010 bis 01.09.2016 wurden in der berichtenden Klinik 68 konsekutive Patienten (NA-Gruppe) an 86 Leisten-
NA nach vorherigen gefäßchirurgischen Operationen behandelt.

Die NAOR-Gruppe umfasste $55 \mathrm{~Pa}$ tienten mit 72 NA und in der NAMRGruppe befanden sich 13 Patienten mit insgesamt 14 NA.

\section{Geschlechtsverteilung und Alter}

In der NA-Gruppe waren mit 92,6\% überwiegend männliche Patienten betroffen $(n=63)$. Zum Zeitpunkt der
Erstsanierung (die erste NA-Operation) lag das Alter durchschnittlich bei 65,5 (Streubreite: 43-87; Standardabweichung [SD]: 10,9) Jahren. Bei Männern war das Durchschnittsalter 65,2 (Streubreite: 43-83; SD: 10,4) Jahre und bei Frauen 69,4 (Streubreite: 48-87, SD: 17,9) Jahre. Ein signifikanter Altersunterschied zwischen Männern und Frauen bestand nicht $(p=0,568)$. 


\begin{tabular}{|c|c|c|}
\hline Voroperationen & $\begin{array}{l}\text { Anzahl der } \\
\text { NA }\end{array}$ & $\begin{array}{l}\text { Angabe in } \\
\%\end{array}$ \\
\hline Bifurkationsprothese aortobifemoral & 45 & 52,3 \\
\hline Patchplastik Leiste & 17 & 19,8 \\
\hline Bypass iliakofemoral (Prothese) & 3 & 3,5 \\
\hline Bypass femoropopliteal P1 (Prothese) & 3 & 3,5 \\
\hline Bypass femorofemoral „cross-over" (Prothese) & 3 & 3,5 \\
\hline Bypass aortofemoral (Prothese) & 2 & 2,3 \\
\hline Interponat bei Femoralaneurysma & 2 & 2,3 \\
\hline Bypass femoropopliteal P3 (Prothese) & 2 & 2,3 \\
\hline $\begin{array}{l}\text { Bifurkationsprothese aortobifemoral/Bypass femoropopliteal P1 } \\
\text { (Prothese) }\end{array}$ & 2 & 2,3 \\
\hline $\begin{array}{l}\text { Bifurkationsprothese aortobifemoral/Bypass femorofemoral "cross } \\
\text { over" (Prothese) }\end{array}$ & 2 & 2,3 \\
\hline Bifurkationsprothese (Anschluss links: AF, rechts: AIC) & 1 & 1,2 \\
\hline Bypass femorokrural/Patchplastik Leiste & 1 & 1,2 \\
\hline Bypass femorokrural auf ATA (Composite-Bypass) & 1 & 1,2 \\
\hline Bypass femoropopliteal P1 (Prothese)/Patch Leiste & 1 & 1,2 \\
\hline Aneurysmorrhaphie mit Patch bei Aneurysma der AFC & 1 & 1,2 \\
\hline Gesamt & 86 & 100,0 \\
\hline
\end{tabular}

\begin{tabular}{|c|c|c|c|c|c|c|}
\hline $\begin{array}{l}\text { Durchführung einer } \\
\text { Leisten-TEA }\end{array}$ & $\begin{array}{l}\text { Mittelwert } \\
\text { (Monate) }\end{array}$ & $\begin{array}{l}\text { SA } \\
\text { (Monate) }\end{array}$ & $\begin{array}{l}\text { Median } \\
\text { (Monate) }\end{array}$ & $\begin{array}{l}\text { Min } \\
\text { (Monate) }\end{array}$ & $\begin{array}{l}\text { Max } \\
\text { (Monate) }\end{array}$ & $\begin{array}{l}p \text {-TEA } \\
\text { ja/nein }\end{array}$ \\
\hline TEA ja & 81,3 & 36,6 & 74 & 4 & 208 & \multirow[t]{3}{*}{$p=0,325$} \\
\hline TEA nein & 72,5 & 36,2 & 65 & 31 & 156 & \\
\hline TEA unbekannt & 158,5 & 25,8 & 160,5 & 131 & 182 & \\
\hline
\end{tabular}

\section{Komorbidität}

Die Patienten hatten eine Reihe von $\mathrm{Ne}$ bendiagnosen, die in (- Tab. 2) aufgeführt werden.

\section{Voroperation}

Die Anlage einer aortobifemoralen Bifurkationsprothese war am häufigsten mit einem NA assoziiert $(n=45$; $52,3 \%)$. Sämtliche Voroperationen sind in - Tab. 3 dargestellt.

\section{Nahtaneurysmaentwicklung bei Zustand nach Anlage einer aortobifemoralen Bifurkationsprothese}

Über $50 \%$ der NA entwickelten sich 85 Monate nach Anlage einer aortobi- femoralen Bifurkationsprothese (siehe - Abb. 1).

Nahtaneurysmaentwicklung in der Leiste und Durchführung einer TEA

Das durchschnittliche Zeitintervall von der primären OP bis zur NA-Entwicklung beträgt 55,4 (Streubreite: 21-101; Median: 49,5; SD: 22,3) Monate und von der NA-OP und Entwicklung eines Rezidiv-NA 51,6 (Streubreite: 4-120; Median: 41,5; SD: 40,4) Monate. Ein signifikanter zeitlicher Unterschied zwischen NA-Entwicklung und Entwicklung eines Rezidiv-NA findet sich nicht $(p=0,683)$. Auch hatte die TEA hier keinen Einfluss $(p=0,325)$ ( $\bullet$ Tab. 4.). Die TEA erfolgte bei 68 Voroperationen $(79,1 \%)$. In 13 Fällen $(15,1 \%)$ war dies während der Operation nicht notwendig. Bei 5 Vorope-

rationen $(5,8 \%)$ war die Durchführung einer TEA nicht bekannt (Patienten in auswärtiger Klinik voroperiert bzw. kein OP-Bericht vorhanden). Der Einfluss einer postoperativen Infektion wurde aufgrund der geringen Fallzahl ( $n=4 ; 4,7 \%)$ sinnvollerweise nicht untersucht.

\section{Symptomatik}

Die anamnestisch angegebene Größenzunahme eines pulsierenden Tumors wurde bei $68,6 \%$ der NA im Operationsnarbenbereich dokumentiert. 15,1\% der NA fielen indirekt durch eine kritische Ischämie und 7,0\% durch eine Verschlechterung der Gehstrecke auf. Bei 5,8\% der NA kam es zu einer gedeckten Ruptur. Zwei NA (2,3\%) waren symptomfreie Zufallsbefunde. In einem Fall $(1,2 \%)$ führte eine Ruptur mit Wunddehiszenz und mit hämorrhagischem Schock zur Diagnose (• Abb. 2).

\section{Querdurchmesser der \\ Leistennahtaneurysmen}

Die Leisten-NA wiesen eine durchschnittliche laterolaterale Größe von 46,4 (Streubreite: 22-110; Median: 40; SD: 22,9) $\mathrm{mm}$ auf. Die Rezidive lagen bei 54,5 (Streubreite: 26-120; Median: 42; SD: $28,9) \mathrm{mm}$. Dieser Unterschied war statistisch nicht signifikant $(p=0,34)$.

\section{Versorgung}

Über die Hälfte der NA $(n=44 ; 51,2 \%)$ wurden primär mittels Interponat versorgt ( $n=42$ in der NAOR-Gruppe und $n=2$ in der NAMR-Gruppe). Bei $25 \mathrm{NA}$ $(29,1 \%)$ wurde eine Aneurysmorrhaphie mit Patch ( $n=19$ in der NAOR-Gruppe und $n=6$ in der NAMR-Gruppe) und bei 17 NA (19,8\%) eine Aneurysmorrhaphie durchgeführt $(n=11$ in der NAORGruppe und $n=6$ in der NAMR-Gruppe). Hierbei wurden in der NAMR-Gruppe das erstaufgetretene NA und nicht das Rezidiv-NA ausgewertet. Bei der Versorgung der Rezidiv-NA war das Interponat die bevorzugte Therapiemethode $(n=12$, $85,7 \%)$. Rhaphie mit und ohne Patch wurde jeweils in einem Fall verwendet $(7,1 \%)$. Die primäre NA-Versorgung erfolgte in 72 Fällen (83,7\%) elektiv. $10 \mathrm{NA}$ 


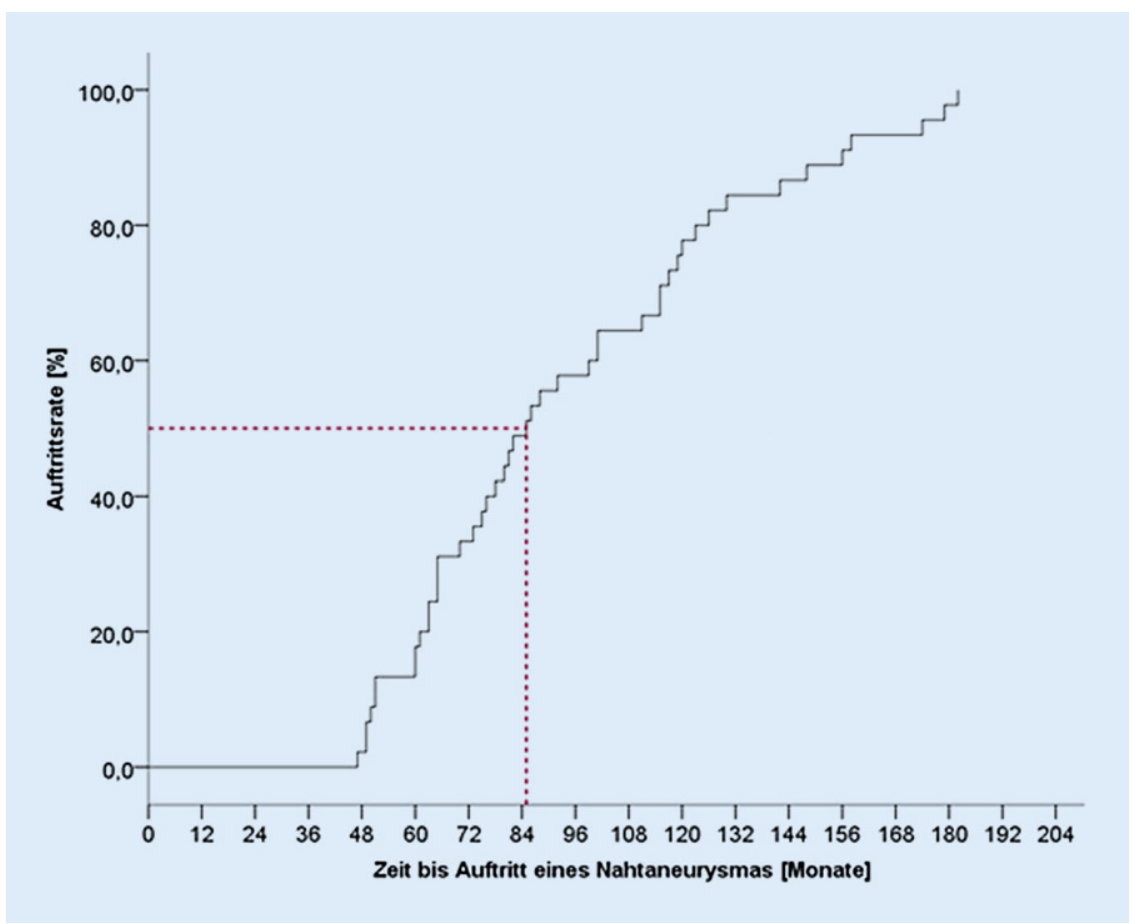

Abb. 1 \ Zeitliches Auftreten der Leisten-NA bei Zustand nach Anlage einer aortobifemoralen Bifurkationsprothese (Kaplan-Meier-Schätzung) $-n_{\text {NA. }}=45$

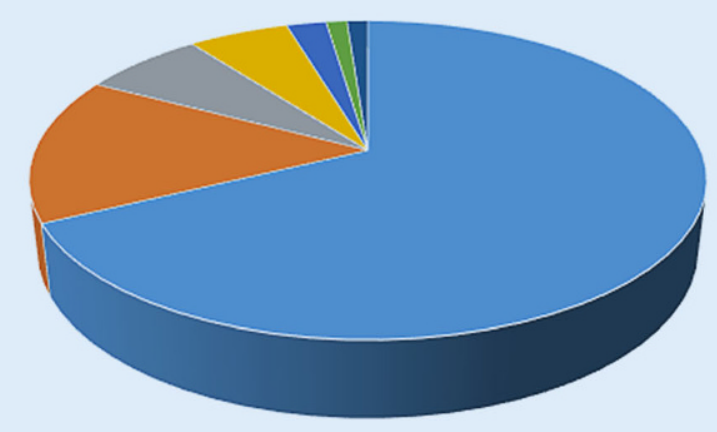
- Größenprogress
= AVK-Verschlechterung
n asymptomatisch
- gedeckte Ruptur mit Hautnekrose

Abb. 2 \ Spektrum der Nahtaneurysmasymptomatik mit relativer Häufigkeit. AVK periphere arterielle Verschlusskrankheit

$(11,6 \%)$ wurden notfallmäßig versorgt und viermal $(4,7 \%)$ wurde eine dringliche Indikation gestellt.

\section{Vergleich der Operations- methoden}

Die Aneurysmorrhaphie hat eine signifikant $(p=0,006)$ 11,45-fach höhere Wahrscheinlichkeit für ein NA-Rezi-

\section{Primäre Offenheitsrate der Leistenrekonstruktionen}

Insgesamt wiesen 35 Interponate bei einer medianen Nachbeobachtungszeit von 29 Monaten eine primäre Offenheitsrate der Gefäßrekonstruktion von 90,6\% nach 60 Monaten auf. Auf der anderen Seite bestand eine primäre Offenheitsrate von 21 Aneurysmorrhaphien mit und ohne Patch zu 33,2\% nach 60 (mediane Nachbeobachtungszeit von 71) Monaten. Die Anwendung von Interponaten und Aneurysmorrhaphien zusammen zeigte eine primäre Offenheit nach 60 (mediane Nachbeobachtungszeit von 32) Monaten von $61,5 \%$. Eine Übersicht wird in Abb. 3 dargestellt. Es zeigte sich ein signifikanter Unterschied bei der primären Offenheitsrate der Gefäßrekonstruktion zwischen Interponaten und Aneurysmorrhaphien $(p=0,001)$.

\section{Postoperative Komplikationen nach der Nahtaneurysma- versorgung}

Es wurden Komplikationen nach der NAErstsanierung in $n=86$ Fällen ausgewertet. Im postoperativen Verlauf hatte es bei fast der Hälfte der NA-Versorgungsoperationen $(n=42 ; 48,9 \%)$ keine Komplikationen gegeben. Die häufigste Komplikation war eine nicht operationspflichtige Lymphozele $(n=22 ; 25,6 \%)$. Ein Hämatom mit erforderlicher Ausräumung war bei $8,1 \%$ der NA zu eruieren $(n=7)$. Eine postoperative Infektion mit Explantation des alloplastischen Materials war in 4 Fällen $(4,7 \%) \mathrm{zu}$ verzeichnen.

Bei 3 Patienten erfolgte eine autologe Rekonstruktion. In einem Fall wurde aufgrund der kompensierten Beinischämie mit einem zusätzlichen chronischen Verschluss des Bifurkationsschenkels von einer Revaskularisation Abstand genommen. Eine Lymphozele mit erforderlicher operativer Revision bzw. eine postoperative Wundheilungsstörung mit notwendiger Wundrevision ist jeweils in 3 Fällen (3,5\%) aufgetreten. Zwei Patienten (2,3\%) erlitten einen akuten Myokardinfarkt (AMI) und jeweils ein Patient (1,2\%) entwickelte eine postoperative Pneumonie mit antibiotikaassoziierte Diarrhoe bzw. einen 
Tab. 5 Repräsentative Übersicht der testend-statistisch vorgenommenen Vergleiche

\begin{tabular}{|c|c|c|}
\hline Auswertungs-/Vergleichsansatz & $\begin{array}{l}\text { Kein signifi- } \\
\text { kanter Unter- } \\
\text { schied }\end{array}$ & $\begin{array}{l}\text { Signifikan- } \\
\text { ter Unter- } \\
\text { schied }\end{array}$ \\
\hline $\begin{array}{l}\text { Altersunterschied zwischen Männern/Frauen bei NA-OP } \\
\text { (Mann-Whitney-U-Test) }\end{array}$ & $p=0,568$ & - \\
\hline $\begin{array}{l}\text { Zeitintervall bis zur NA-Entwicklung und Entwicklung eines Rezi- } \\
\text { divs in der Leistenregion (Wilcoxon-Test) }\end{array}$ & $p=0,683$ & - \\
\hline $\begin{array}{l}\text { Zeitintervall bis zur NA-Entwicklung nach einer Primäroperation } \\
\text { in der Leistenregion mit/ohne TEA } \\
\text { (Mann-Whitney-U-Test) }\end{array}$ & $p=0,325$ & - \\
\hline $\begin{array}{l}\text { Durchmessergröße in der Leiste bei NA und Rezidiv-NA } \\
\text { (Wilcoxon-Test) }\end{array}$ & $p=0,34$ & - \\
\hline $\begin{array}{l}\text { Wahrscheinlichkeit einer postoperativen Komplikation bei notfall- } \\
\text { mäßiger Versorgung des NA im Vergleich zu dringlicher/elektiver } \\
\text { Versorgung ( } x^{2} \text { nach Pearson) }\end{array}$ & $p=0,09$ & - \\
\hline $\begin{array}{l}\text { Wahrscheinlichkeit eines Rezidivs bei Aneurysmorrhaphie ohne } \\
\text { Patch im Vergleich zum Interponat ( } x^{2} \text { nach Pearson) }\end{array}$ & - & $p=0,006$ \\
\hline $\begin{array}{l}\text { Wahrscheinlichkeit eines Rezidivs bei Aneurysmorrhaphie mit } \\
\text { Patch im Vergleich zum Interponat ( } x^{2} \text { nach Pearson) }\end{array}$ & - & $p=0,026$ \\
\hline $\begin{array}{l}\text { Primäre Offenheitsrate in der Leistenregion zwischen Interpona- } \\
\text { ten und Aneurysmorrhaphien (Log-Rank-Test) }\end{array}$ & - & $p=0,001$ \\
\hline
\end{tabular}

Verschluss mit notwendiger Thrombektomie (Mehrfachnennungen möglich).

Die 30-Tage-Letalität betrug 2,3\% $(n=2$ aus der NAOR-Gruppe, $n=0$ aus der NAMR-Gruppe). Ein Patient verstarb durch einen AMI und der weitere Patient durch eine kardiopulmonale Dekompensation bei einer zusätzlichen hämatoonkologischen Grunderkrankung. Ein signifikanter Unterschied beim Auftreten von postoperativen Komplikationen bezüglich der Indikationsstellung (elektiv/dringlich $v s$. Notfall) war nicht zu eruieren $(p=0,09)(\bullet$ Tab. 5).

\section{Diskussion}

NA sind mittlerweile mit dem optimierten periinterventionellen/-operativen Management, insbesondere hinsichtlich des Erreichens eines ansprechenden mittel- bis langfristigen Ergebnisses, insbesondere Überlebens nach gefäßchirurgischen Rekonstruktionen ein sehr relevanter Aspekt einer anliegenden Sekundärversorgung geworden und daher zunehmend in den Blickpunkt der klinischen Routineversorgung gerückt. Eine eigene und seltene Entität bilden die "Graft“-Aneurysmen (echtes Prothesenaneurysma), die von einem NA (falsches Aneurysma) mit Lokalisation im Bereich der Anastomosenstelle abzugrenzen sind [1]. Als primäre Diagnostik der NA wurde häufig die Duplexsonographie im ambulanten Bereich verwendet. Bei einem duplexsonographischen Nachweis eines NA wurde präoperativ die CTA durchgeführt. Die MRA erfolgte vorwiegend ambulant bei Patienten mit einer Stadienverschlechterung der PAVK, wobei das NA als Zufallsbefund dargestellt wurde. Falls im Rahmen einer endovaskulären Versorgung ein LeistenNA angiografisch zufällig diagnostiziert wurde, erfolgte im Verlauf eine offenchirurgische Sanierung. Die DAS ist für die NA-Diagnostik aufgrund der partiellen oder kompletten Thrombosierung der Nahtaneurysmen ungeeignet.

Die erhobenen demografischen Daten sind mit anderen Studien vergleichbar, in denen der männliche Anteil mit 80 bis $93 \%$ und das Durchschnittsalter mit 62,8 bis 66,4 Jahren angegeben wurde $[6,9,24]$.

Dass hinsichtlich der Komorbidität des Patientenkollektivs vorwiegend $\mathrm{Pa}$ tienten mit aHT, PAVK, Nikotinabusus und KHK vertreten sind, begründet sich durch das atherogene Risikoprofil des Patientenkollektivs [12]. Bei 14,7\% der Patienten bestand eine orale Antikoagulation (OAK: Vitamin-K-Antagonisten und neue Antikoagulantien). In dem präsentierten Patientenklientel wurde die kardiologisch bzw. gefäßchirurgischerseits indizierte Antikoagulation postope- rativ fortgeführt. Bei einer zusätzlichen intraoperativen Stentimplantation oder Anlage eines infragenualen Prothesenbypasses erhielten die Patienten eine duale Thrombozytenaggregationshemmer(TAH)-Therapie. Nach durchgeführter Venenbypassanlage erfolgte die Einstellung auf Falithrom. In den restlichen Fällen war eine TAH-Monotherapie, falls kardiologischerseits nicht anders indiziert, ausreichend. Eine zu intensive postoperative Antikoagulation kann allerdings die Einheilung der Prothese behindern, fördert die Entwicklung einer Lymphfistel und begünstigt eine Aneurysmabildung [15, 20]. Hernienerkrankungen wurden bei $16,2 \%$ der Patienten nachgewiesen. Diese lässt sich („grob-klinisch“) als Ausdruck einer Gewebeschwäche deuten [17], wobei sich formal damit keine wirkliche Risikoerhöhung im Vergleich zur Lebensprävalenz der Allgemeinbevölkerung ausmachen lässt. In 14,7\% der Fälle war auch ein AAA diagnostiziert. Dahingehend wäre zukünftig eine histologische Untersuchung mit Biomarkern der betroffenen arteriellen Gefäßwand sinnvollerweise in weiterführenden wissenschaftlichen Studienschritten zu erwägen, um eine definitive Gewebeschwäche aufzudecken $[11,16,18]$.

$11,8 \%$ der Patienten litten an einem DM, wobei eine Korrelation zwischen DM und NA in der Studie von Schellack et al. nicht bestätigt wurde [26].

Ein genauer Vergleich zu anderen Studien ist aufgrund eines unterschiedlichen Studiendesigns und der jeweiligen $\mathrm{He}$ terogenität des Patientenkollektivs nicht erschöpfend und verlässlich möglich. In der Studie von Mulder et al. wiesen anamnestisch $47 \%$ der Patienten eine aHT, 24,4\% einen Myokardinfarkt und 22\% eine COPD auf. $18 \%$ der Patienten litten an einer chronischen Niereninsuffizienz und $14 \%$ zeigten eine zerebrovaskuläre Ischämie [22].

Bei der gefäßchirurgischen Primäroperation sind die meisten NA nach Anlage einer aortobifemoralen Bifurkationsprothese $(n=45 ; 52,3 \%)$ und A.-femoralisPatchplastik $(n=17 ; 19,8 \%)$ aufgetreten. In verschiedenen Studien gehörte die Anlage eines aortobifemoralen Bypasses zu 


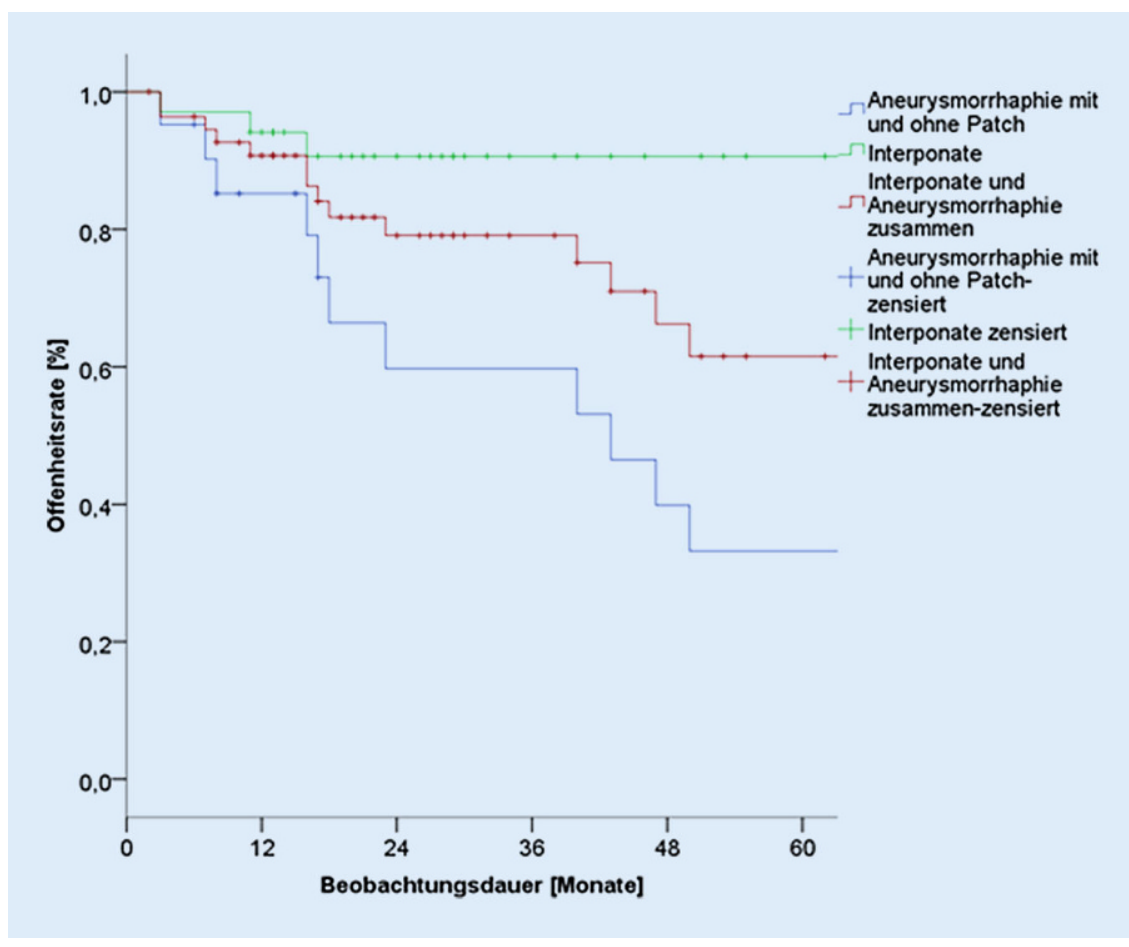

Abb. $3 \Delta$ Durchgängigkeitsraten der Leistengefäßrekonstruktionen bei Nahtaneurysmen mit signifikantem Unterschied zwischen Interponaten (grünerGraph) und Aneurysmorrhaphien (blauer Graph). $p=0,001$

den häufigsten Voroperationen $[7,9,19]$. Die hohe Inzidenz wurde durch

- die Lokalisation im Beugungssegment (Leistenbereich),

- das Vorhandensein einer gelenküberschreitenden Anastomose (sowie)

- die Verwendung von Polyester mit hoher Dilatationstendenz

erklärt.

In der Literatur ist die Durchführung einer TEA als Risikofaktorzur Entstehung von NA beschrieben [3, 9]. In der vorliegenden Arbeit konnte dieser Aspekt nicht nachgewiesen werden. Die Schlussfolgerung wäre, dass die Schwäche der Gefäßwand relevanter im Hinblick auf die Entwicklung eines NA ist.

In der Literaturrecherche erstrecken sich die Zeitintervalle von der Primäroperation bis zur (Rezidiv-)NA-Entwicklung von 2,1 bis auf 15 Jahre, was sich mit den eigenen Ergebnissen deckt [7, 9, 13, 27]. Die NA im aortalen und iliakalen Bereich entstehen nach einem längeren Zeitraum [20]. Die Zeitintervalle bei der Entstehung der Rezidiv-NA sind sowohl in der Literatur als auch in der eigenen klinischen Untersuchung kürzer. Dies lässt sich durch eine vorgeschädigte Gefäßwand im Rahmen der Voroperation begründen. Allerdings erwies sich das Zeitintervall in der eigenen Patientengruppe als nicht signifikant unterschiedlich. Tritt eine postoperative Infektion auf, ist das Zeitintervall ebenfalls kürzer [6, 10]. Debus et al. weisen darauf hin, die Nahtrupturen im Rahmen einer Infektion differenzialdiagnostisch abzugrenzen [5].

Hinsichtlich der Symptomatologie gehört der pulsierende Tumor in der Literatur zu den häufigsten Symptomen der NA. Andere Anzeichen sind mit unterschiedlicher Inzidenz beschrieben worden, erklärt durch die unterschiedlichen Patientenkollektive. Eine Schmerzangabe als Symptom war seitens der Patienten sehr unterschiedlich, teilweise nicht NAspezifisch angegeben („Schmerzen im Bein"), sodass dies nicht in der Symptomatik ausgewertet wurde. Demarche et al. beschreiben einen schmerzfreien pulsierenden Tumor in $64,1 \%$ der Fälle sowie eine Ischämierate von $19 \%$ [6]. Einen schmerzhaften Tumor zeigten 8,5\% der Fälle. Die Blutungskomplikation lag bei $7 \%$. Ein Ödem bzw. eine Mikroembolie wurde jeweils bei $0,7 \%$ der
NA diagnostiziert. Im Patientenkollektiv von Marković et al. betrug die Infektionsrate $24,7 \%$ mit einer resultierenden Blutungskomplikationsrate von $29,9 \%$ [19]. Die genannten wissenschaftlichen Ergebnisse unterstreichen dabei deutlich die Bedeutung eines wirksamen postoperativen Dispensaires (fachspezifische klinische und bildgebende Verlaufskontrolle).

Hinsichtlich des Querdurchmessers der Leisten-NA waren die Rezidiv-NA in der laterolateralen Messung gröBer im Vergleich zu den primären NA $(54,4$ vs. $46,4 \mathrm{~mm})$. Der Unterschied im Durchmesser war statistisch jedoch nicht signifikant $(p=0,34)$. Nach Recherche der Fachliteratur ist eine derartige Auswertung des NA-Durchmessers noch nicht beschrieben worden. Dies lässt sich eventuell durch eine mangelnde Compliance der Patienten erklären, die das Krankheitsbild von der ersten NAVersorgung zwar kennen, sich aber dennoch erst im fortgeschrittenen Stadium des Rezidivs gefäßchirurgisch vorstellen. Als weitere Ursache ließe sich ein insuffizientes Dispensaire diskutieren.

Hinsichtlich der Versorgung der Primär-NA wurden alle NA im Bereich der Leiste operativ therapiert. Die Leistenregion als Beugungssegment bleibt weiterhin eine Domäne der offenen Chirurgie mit guter Offenheitsrate [30]. In den anderen anatomischen Regionen gewinnt die endovaskuläre Behandlung bei geeigneter Anatomie zunehmend an Bedeutung $[2,13,28,31]$. Eine Thrombininjektion im Rahmen der NA-Versorgung ist in der Literatur nur in Einzelfällen beschrieben [23].

Dennis et al. berichtete von einer Offenheitsrate von $97,5 \%$ bei einem durchschnittlichen Follow-up von 2,4 Jahren [7]. Im eigenen Patientenkollektiv betrug in der Leistenregion die primäre Offenheitsrate von Interponaten 90,6\% und von Aneurysmorrhaphien mit und ohne Patch 33,2\% nach 5 Jahren. Die deutlich schlechtere primäre Offenheitsrate der Aneurysmorrhaphien war vorwiegend durch die Notwendigkeit einer operativen Revision bei NA-Progress und nicht aufgrund des Verschlusses bedingt.

Die unterschiedliche Beobachtungsdauer bei den Patienten in der Studie wur- 

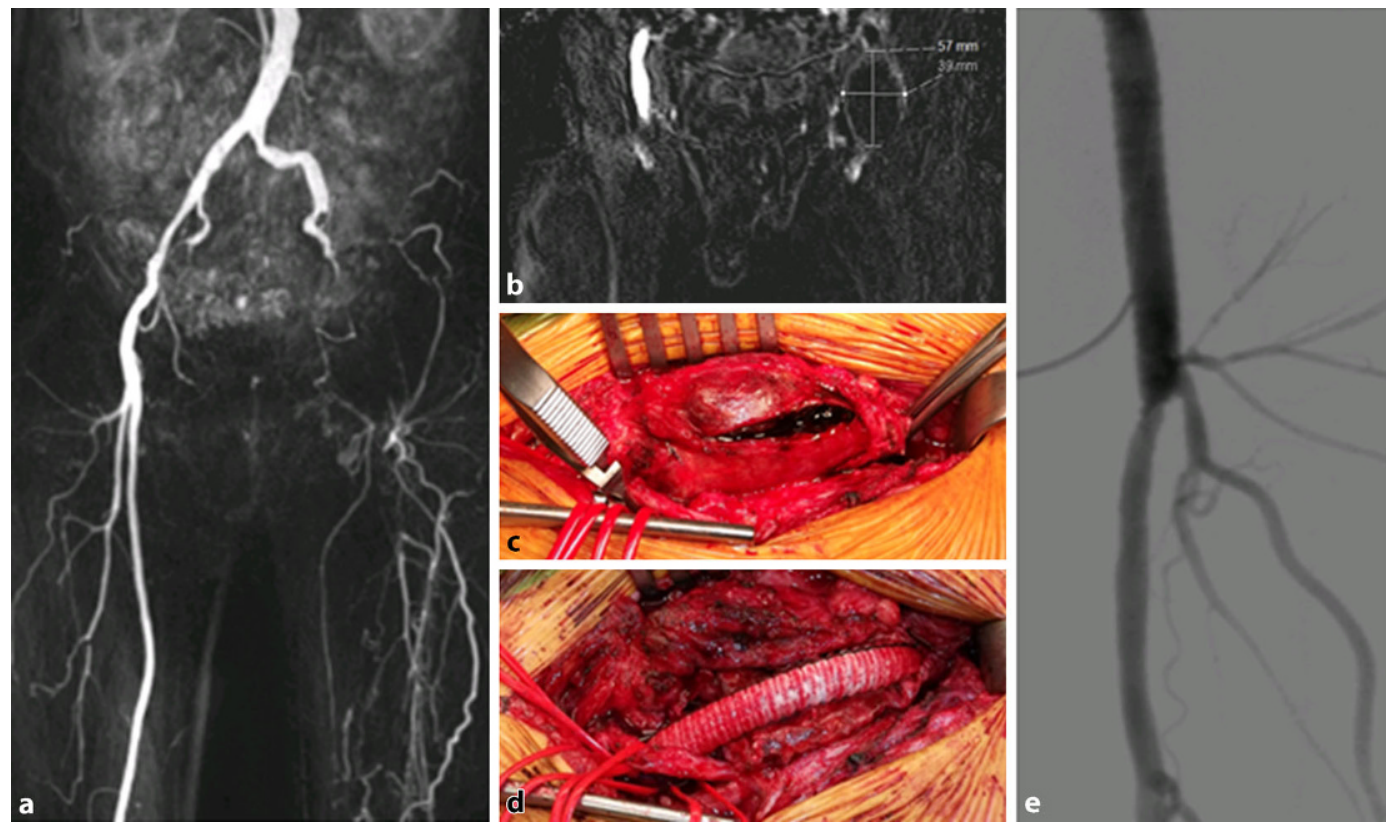

Abb. 4 A Beispielhafte Bildgebung bei einem repräsentativen Fall (klinisch: inkomplette Beinischämie links). a MR-Angiographie der Becken/Beingefäße - Diagnose: Verschluss der A. iliaca externa links bei einem komplett thrombosierten NA der A. femoralis communis mit Thrombosierung der Äste der A. profunda femoris, chronischer Verschluss der A. femoralis superficialis links (anamnestisch:Z. n. TEAund Patchplastik der A. femoralis communis links, klinisch:inkomplette Beinischämie links). b Darstellung und Ausmessung des komplett thrombosierten NA der A. femoralis communis links. c Ausklemmung der Gefäße kranial und kaudal des NA, Eröffnung des komplett thrombosierten NA der linken Leiste. - Sämtliche Äste der A. profunda femoris links sind mit einem Loop angezügelt. $\mathbf{d}$ Ansicht nach Resektion der NA-Interponatanlage femoroprofundal links (Prothese: Ø: $7 \mathrm{~mm}$; FlowNit Bioseal, Fa. Jotec $\mathrm{GmbH}$, Hechingen). - Sämtliche Äste der A. profunda femoris und der N. femoralis sind mit einem Loop angezügelt. e Intraoperative Kontroll-DSA mit offenem Interponat und offenen Ästen der A. profunda femoris links nach durchgeführter Thrombektomie derselben

de durch lückenhafte regelmäßige Nachsorge in der gefäßchirurgischen Nachsorge verursacht.

In der Leiste findet eine endovaskuläre Behandlung nur in Ausnahmefällen und bei multimorbiden Patienten statt [8].

Hinsichtlich der Versorgung der Primär-NA ist in der Literatur eine Rezidivrate von $8,5 \%$ bis $10,4 \%$ beschrieben $[6,21,24]$. Eine Interponatanlage ist die Methode der Wahl im Rahmen der gefäßchirurgischen Rekonstruktion ([6, 9, 10, 20]; - Abb. 4). Die erhöhte Rezidivrate bei der Aneurysmorrhaphie mit oder ohne Patch lässt sich am ehesten durch partielles/residuelles Belassen der aneurysmatisch veränderten Gefäßwand basisnah erklären.

Hinsichtlich der postoperativen Komplikationen und der Mortalität nach der NA-Versorgung stellte eine nicht operationspflichtige Lymphozele die häufigste postoperative Komplikation dar (25,6\%). Dies erklärt sich durch den Revisionseingriff im vernarbten Gewebe und der assoziierten Zerstörung von Lymphbahnen.
2,3\% der Patienten verstarben innerhalb von 30 Tagen nach der Operation. In der Literatur ist eine operative Mortalität von $2,7 \%$ bis 7,6\% beschrieben. Ein Infektnachweis erhöht die postoperative Morbidität und Mortalität [6, 22, 24]. Mulder et al. beschrieben in ihrem Kollektiv von 135 Patienten mit 158 nicht infizierten NA nach aortoiliakalen und aortofemoralen Rekonstruktionen eine perioperative Morbidität von $17 \%$. Hierbei stellte sich die intraoperative Blutung vorwiegend bei den Notfalleingriffen als die häufigste Komplikation dar [22]. Die Patienten mit aortaler (31\%), iliakaler (38\%) und femoraler (31\%) Lokalisation des NA wurden zwischen 1963 und 1990 operiert, was auch die erhöhte Morbidität erklärt. Die Letalität und Komplikationsrate der NA-Versorgung sind in der Fachliteratur höher im Vergleich zur Primäroperation beschrieben. Ebenfalls weist die notfallmäßige im Vergleich zur elektiven Versorgung eine erhöhte Morbidität und Letalität auf [20]. In der vorgelegten Studie wurde allerdings kein sig- nifikanter Unterschied zwischen elektiv/ dringlich $v s$. Notfall-OP bzgl. der postoperativen Komplikationsrate festgestellt $(p=0,09)$. Im Vergleich zur Studie von Mulder et al. wurden im eigenen Patientenkollektiv nur Leisten-NA ausgewertet. Weiterhin traten alle nicht operationspflichtige Lymphozelen nach elektiven Eingriffen auf, was auch zu diesem nichtsignifikanten Unterschied geführt hat.

Als Limitation konnte aufgrund der retrospektiven Auswertung des Patientenkollektivs eine klare Definition der sicheren Risikofaktoren für die NA-Manifestation nicht verlässlich bestimmt werden. Eine multivariate Analyse war über die univariate Analyse der deskriptivstatistisch eruierten und statistisch verglichenen Einflussfaktoren hinaus nicht möglich, da eine Kontrollgruppe ähnlichen gefäßchirurgischen Eingriffsprofils fehlte und die diagnosespezifisch ausgerichtete konsekutive Patientenkohorte des postoperativ entstandenen NA im Vordergrund stand. Die laterolaterale Durchmesserbestimmung wurde durch 
drei verschiedene, aber komplementär aufzufassende diagnostische Verfahren erhoben (Duplexsonographie, CTA und MRA), wobei die Messung vom jeweiligen Untersucher (Gefäßchirurg, Radiologe) abhängig war.

\section{Zusammenfassung und Schlussfolgerung}

Trotz Entwicklung des Gefäßersatz- und Nahtmaterials sind die NA mit einer Inzidenz von $0,2-15 \%$ keine seltene Komplikation in der Gefäßchirurgie. Die meisten NA im eigenen Patientenkollektiv traten nach Anlage einer aortobifemoralen Prothese (52,3\%) auf. Das durchschnittliche Zeitintervall bis zur NA-Entwicklung in der Leiste betrug 55,4 Monate und 51,6 Monate bis zur Entwicklung eines Rezidivs. Ein signifikanter zeitlicher Unterschied bis zur Entwicklung eines NA bzw. eines Rezidiv-NA ließ sich nicht bestätigen $(p=0,683)$. Obwohl die TEA in der Literatur als Risikofaktor für die NAEntwicklung angegeben ist, konnte im präsentierten Patientenkollektiv kein signifikanter zeitlicher Unterschied nachgewiesen werden $(p=0,325)$. Der durchschnittliche Durchmesser der LeistenNA betrug 46,4 mm und der Rezidiv-NA in der Leiste $54,5 \mathrm{~mm}$, ohne dass sich ein signifikanter Unterschied zwischen den beiden Gruppen $(p=0,34)$ herausstellte. Dies lässt eine Incompliance der Patienten oder ein insuffizientes angiologisches Dispensaire vermuten. Bei der Versorgung von NA gewinnen die interventionellen Techniken dank einer geringeren Komplikationsrate und Invasivität an Bedeutung, wobei die Leistenregion weiterhin eine Domäne der offengefäßchirurgischen Rekonstruktion (Interponatanlage) bleibt. Postoperativ sollen die Patienten über die Wichtigkeit und Empfehlung einer regelmäßigen und lebenslangen gefäßchirurgischen Nachsorge aufgeklärt werden.

\section{Fazit für die Praxis}

- Das Leisten-NA ist eine klinisch relevante Spätkomplikation.

- Das Interponat ist die Therapie der Wahl bei der NA-Revision.
- Die Rekonstruktion mit Rhaphie hat ein hohes Rezidivrisiko.

- Bei Patienten mit Ischämiesymptomatik soll differenzialdiagnostisch auch an ein komplett thrombosiertes NA gedacht werden.

- Es ist bedeutsam, eine lebenslange Nachsorge nach gefäßchirurgischen Eingriffen mit Kontrolle aller Gefäßanastomosen (Stichwort Synchronaneurysmen) zu gewährleisten.

\section{Korrespondenzadresse}

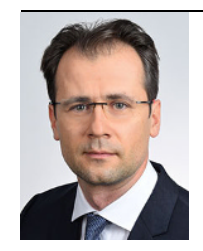

Prof. Dr. F. Meyer

Arbeitsbereich Gefäßchirurgie, Klinik für Allgemein-, Viszeral-, Gefäß- und Transplantationschirurgie, Universitätsklinikum Magdeburg Leipziger Straße 44, 39120 Magdeburg, Deutschland frank.meyer@med.ovgu.de

Funding. Open Access funding enabled and organized by Projekt DEAL.

\section{Einhaltung ethischer Richtlinien}

Interessenkonflikt. P. Romancik, F. Meyer, A. Hribaschek, U. Redlich, Z. Halloul und J. Tautenhahn geben an, dass kein Interessenkonflikt besteht.

Für diesen Beitrag wurden von den Autoren keine Studien an Menschen oder Tieren durchgeführt. Für die aufgeführten Studien gelten die jeweils dort angegebenen ethischen Richtlinien.

Open Access. Dieser Artikel wird unter der Creative Commons Namensnennung 4.0 International Lizenz veröffentlicht, welche die Nutzung, Vervielfältigung, Bearbeitung, Verbreitung und Wiedergabe in jeglichem Medium und Format erlaubt, sofern Sie den/die ursprünglichen Autor(en) und die Quelle ordnungsgemäß nennen, einen Link zur Creative Commons Lizenz beifügen und angeben, ob Änderungen vorgenommen wurden.

Die in diesem Artikel enthaltenen Bilder und sonstiges Drittmaterial unterliegen ebenfalls der genannten Creative Commons Lizenz, sofern sich aus der Abbildungslegende nichts anderes ergibt. Sofern das betreffende Material nicht unter der genannten Creative Commons Lizenz steht und die betreffende Handlung nicht nach gesetzlichen Vorschriften erlaubt ist, ist für die oben aufgeführten Weiterverwendungen des Materials die Einwilligung des jeweiligen Rechteinhabers einzuholen.

Weitere Details zur Lizenz entnehmen Sie bitte der Lizenzinformation auf http://creativecommons.org/ licenses/by/4.0/deed.de.

\section{Literatur}

1. Barth U, Wasseroth K, Meyer F (2018) Graftaneurysmen als Langzeitkomplikation einer Polyesterprothese und deren Management. Chirurg 89:212-221

2. Beck AW, Bos WT, Vourliotakis G et al (2009) Fenestrated and branched endograft repair of juxtarenal aneurysms after previous open aortic reconstruction. JVasc Surg 49:1387-1394

3. Becker HM, Kortmann H (2003) Nahtaneurysmen. In: Heberer G, van Dongen RJAM (Hrsg) Gefäßchirurgie. Springer, Berlin, Heidelberg, S178-184

4. Casey PJ, LaMuraglia GM (2005) Anastomotic aneurysms. In: Rutherford RB (Hrsg) Vascular surgery, 6. Aufl. Elsevier Saunders, , S894-902

5. Debus ES, Diener H (2012) Klinik und offene Revaskularisation. In: Debus ES, Gross-Fengels W (Hrsg) Operative und interventionelle Gefäßmedizin. Springer, Berlin, Heidelberg, S664-678

6. Demarche $M$, Waltregny $D$, van Damme $H$, Limet R (1999) Femoral anastomotic aneurysms: pathogenic factors, clinical presentations and treatment. A study of 142 cases. Cardiovasc Surg 7:315-322

7. Dennis JW, Littooy FN, Greisler HP et al (1986) Anastomotic pseudoaneurysms. A continuing late complication of vascular reconstructive procedures. Arch Surg 121:314-317

8. Derom A, Nout E (2005) Treatment of femoral pseudoaneurysms with endograft in high-risk patients. Eur JVasc Endovasc Surg 30:644-647

9. Di Marzo L, Strandness EL, Schultz RD et al (1987) Reoperation for femoral anastomotic false aneurysm. A 15-year experience. Ann Surg 206:168-172

10. Harnoss BM, Zühlke H, Matura G et al (1989) Das Anastomosenaneurysma. Zentralbl Chir 114:169-174

11. Henriksson AE, Lindqvist $M$, Sihlbom C et al (2018) Identification of potential plasma biomarkers for abdominal aortic aneurysm using tandem mass tag quantitative proteomics. Proteomes 6:E43

12. Hepp W (2007) Risikoabklärung. In: Hepp W, Kogel H (Hrsg) Gefäßchirurgie. Urban \& Fischer / Elsevier, München, S59-62

13. Hyhlik-Dürr A, Bischoff MS, Peters AS et al (2013) Endovascular therapy of para-anastomotic aneurysms of the aorta. Technical options. Chirurg 84:881-888

14. Kogel H (2007) Anschlussaneurysmen und Nahtrupturen. In: Hepp W, Kogel H (Hrsg) Gefäßchirurgie. Urban \& Fischer / Elsevier, München, S763-770

15. Kortmann H(2007) Pathophysiologische Ursachen arterieller Aneurysmen. In: Hepp W, Kogel H (Hrsg) Gefäßchirurgie. Urban \& Fischer / Elsevier, München, S245-252

16. Larena-Avellaneda A, Debus ES (2012) Aneurysmatische Gefäßerkrankungen. In:DebusES, GrossFengels W (Hrsg) Operative und interventionelle Gefäßmedizin. Springer, Berlin, Heidelberg, S 129-135

17. Leister I, Becker H(2006) Hernien. In: Siewert (Hrsg) Chirurgie, 8. Aufl. Springer, Heidelberg, S732-746

18. Li T, Jiang B, Li X et al (2018) Serum matrix metalloproteinase- 9 is a valuable biomarker for identification of abdominal and thoracic aortic aneurysm: a case-control study. BMC Cardiovasc Disord 18:202

19. Marković DM, Davidović LB, Kostić DM et al (2007) False anastomotic aneurysms. Vascular 15:141-148 
20. Maxfield KM, Guzman RJ (2014) Local complications: anastomotic aneurysms. In: Cronenwett, Johnston (Hrsg) Rutherford's vascular surgery, 8. Aufl. Elsevier Saunders, , S682-692

21. Melliere D, Becquemin JP, Cervantes-Monteil $F$ et al (1996) Recurrent femoral anastomotic false aneurysms: is long term repair possible? Cardiovasc Surg 4:480-482

22. Mulder EJ, van Bockel JH, Maas J et al (1998) Morbidity and mortality of reconstructive surgery of noninfected false aneurysms detected long after aortic prosthetic reconstruction. Arch Surg 133:45-49

23. Nakamura T(2011) Anastomoticpseudoaneurysm formation after bypass grafting to porcelainized peroneal artery: successful treatment by percutaneous thrombin injection. Ann Vasc Surg 25:980.e3-980.e6

24. Pogorzelski R, Fiszer P, Toutounchi S et al (2013) Anastomotic aneurysms - 20-years of experience from one center. Pol Przegl Chir 85:181-191

25. Richardson JV, Mcdowell HA (1976) Anastomotic aneurysms following arterial grafting: a 10-year experience. Ann Surg 184:179-182

26. Schellack J, Salam A, Abouzeid MA et al (1987) Femoral anastomotic aneurysms: a continuing challenge. JVasc Surg 6:308-317

27. Stoney RJ, Albo RJ, Wylie EJ (1965) False aneurysms occuring after arterial grafting operations. Am J Surg 110:153-161

28. Verhoeven EL, Muhs BE, Zeebregts CJ et al (2007) Fenestrated and branched stent-grafting after previous surgery provides a good alternative to open redo surgery. Eur J Vasc Endovasc Surg 33:84-90

29. Vollmar JF (1996) Anastomosen-(„Naht"-)aneurysma. In: Komplikationen bei rekonstruktiven Eingriffen am Arteriensystem. Thieme, Stuttgart, S 419-422

30. Waibel P (1994) Falsche Aneurysmen nach Rekonstruktion wegen PAVK. Beobachtungen über 15 bis 25 Jahre. Vasa 23:43-51

31. Wu Z, Xu L, Raithel D et al (2016) Endovascular repair of proximal para-anastomotic aneurysms after previous open abdominal aortic aneurysm reconstruction. Vascular24:227-232

\section{Entscheidungsassistenz}

Eine Brücke auf dem Weg zur Einwilligungsfähigkeit von Menschen mit Demenz in medizinische Maßnahmen

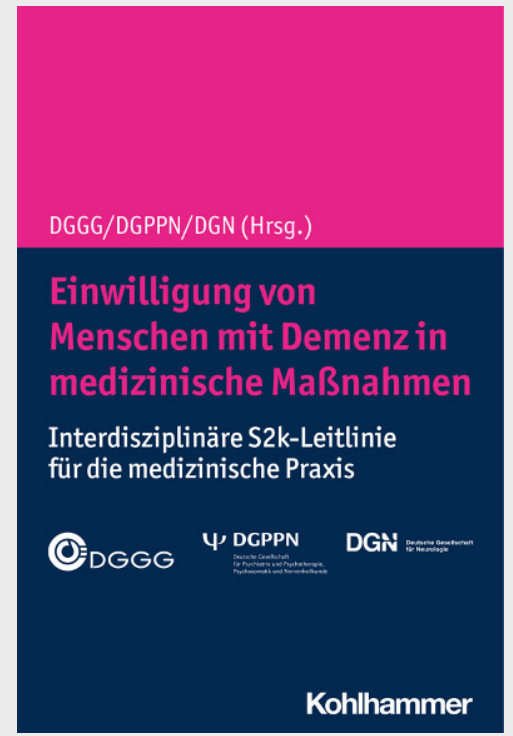

Die 2020 veröffentlichte Interdisziplinäre Leitlinie „Einwilligung von Menschen mit Demenz in medizinische Maßnahmen" [1] und die 2021 hierzu in der Zeitschrift für Gerontologie+Geriatrie CME-validierte Fortbildung [2] zeigen einen strukturierten Handlungspfad mit dem Ziel, die Selbstbestimmung des Menschen mit Demenz möglichst lange zu erhalten.

Die Diagnose einer Demenz kann und darf nicht mit Unfähigkeit zur Einwilligung gleichgesetzt werden. Die Entscheidung des Patienten für eine medizinische Maßnahme muss auf einer informierten Einwilligung beruhen Die Voraussetzungen hierfür sind:

1. Informationsvermittlung

2. Informationsverständnis

3. freie Entscheidung

4. Einwilligungsfähigkeit

Bestehen Zweifel an der Einwilligungsfähigkeit und somit an der Bestimmbarkeit des Willens eines Menschen mit Demenz, muss die Einwilligungsfähigkeit im Sinne eines klinischen Urteils valide geprüft werden: 1. Informationsverständnis

2. Einsicht in die Krankheit und die Notwendigkeit der Behandlung

3. Urteilsvermögen

4. Kommunizieren einer Entscheidung Die Synthese dieser vier Felder ergibt die Bestimmbarkeit des Willens.
Erscheint die Einwilligungsfähigkeit des Gegenübers in einen medizinischen Eingriff nach valider Prüfung nicht oder nicht sicher gegeben, kann durch Herbeiführen einer so genannten Entscheidungsassistenz unter Umständen eine Einwilligungsfähigkeit erreicht werden.

Entscheidungsassistenz ließe sich als einen empathisch wertschätzenden individualisierten Prozess im Konzept des ärztlichen Aufklärungsgespräches in räumlicher, sozialer und dinglicher Kontextgestaltung definieren: eine ruhige Atmosphäre ohne störende Einflüsse, eine den Besonderheiten der Demenz und etwaigen weiteren Beeinträchtigungen der betreffenden Person angepasste Form der Kommunikation, vor allem das Sehen und das Hören betreffend, gegebenenfalls unter Hinzunahme notwendiger Hilfen und Hilfsmittel, ein klare und einfache bis hin zur leichten Sprache, bei Bedarf eine zusätzliche schriftliche Information, gegebenenfalls auch Anwesenheit einer Begleitperson. Fazit: Auch wenn nicht in allen Fällen bei betroffenen Personen eine Einwilligungsfähigkeit erreicht werden kann, so kann doch mithilfe dieses Konzeptes zwischen einer eher zustimmenden und einer eher ablehnenden Haltung (, assent" versus "dissent") differenziert werden.

\section{Literatur}

[1] DGGG/DGPPN/DGN (Hrsg.) (2020) Einwilligung von Menschen mit Demenz in medizinische Maßnahmen, Interdisziplinäre S2k-Leitlinie für die medizinische Praxis (AWMF-Leitlinie Registriernummer 108-001), 1. Auflage, W. Kohlhammer GmbH, Stuttgart; www.awmf.org/leitlinien/detail/Il/108001.html

[2] Haberstroh J, Tesky VA , Pantel J (2021) Einwilligungsfähigkeit von Menschen mit Demenz, Einblicke in die S2k-AWMF-Leitlinie 108-001. Z Gerontol Geriat 54:167-175; doi.org/10.1007/s00391-020-01820-4

\section{Korrespondenzadresse:}

Dr. Udo Hennighausen

Augenarzt, Anerkennung Geriatrie

Jürgen-Töpfer-Straße 10

22763 Hamburg

E-Mail:Udo. Hennighausen@web.de 